\title{
I TIPI ITALIANI CONESSO, LUNGHESSO, SOVRESSO, SOTTESSO E LA GRAMMATICALIZZAZIONE DI IPSE
}

\section{UNA DESCRIZIONE GRAMMATICALE}

In italiano antico erano presenti delle costruzioni preposizionali in cui il pronome esso appare agglutinato alla base preposizionale semplice. Si tratta dei tipi conesso, lunghesso, sovresso (sopresso), sottesso, che compaiono in testi del '200 e del ' 300 , in particolare in Dante e Boccaccio, e hanno continuatori nella lingua letteraria sino agli inizi del '900 (in Pascoli, D’Annunzio e Pirandello):

\section{Conesso}

Dante, Inferno 32: «conesso un colpo per la man d'Artù»

Boccaccio, Novella VII, 1 «Dell'altre volte ritrovandosi colla donna, molto di questa incantazione rise conesso lei»»

Cavalca, Epist. Eustochio, cap. 2 «Non temere (disse), che più non sono conesso noi, che contro a noi»

Ariosto, Orlando Furioso 40, 55 «Fu Bucifar dell'Algazera morto / Conesso un colpo da Olivier gagliardo» 1

\section{Lunghesso}

Conti morali (ed. Zambrini) 10 «e poscia cercò tanto, ch'elli trovò el santo romito, sotto el muro lunghesso la torre di Dauro»

Dante, Purgatorio 2, v. 10 «Noi eravam lunghesso mare ancora, / come gente che pensa a suo cammino»

Dante, Purgatorio 19, v. 27 »Ancor non era sua bocca richiusa, / quand'una donna apparve santa e presta / lunghesso me per far colei confusa»

Boccaccio, Amorosa Visione, c. 7, v. 10 «Lunghesso Fauno e Pico lor vid'io / seguire, ed il gran Belo dopo loro»

\footnotetext{
* Indirizzo dell'autrice: Facoltà di Lettere e Filosofia, Dipartimento di Filologia Moderna, Via Porta di Massa 1, 80133 Napoli, Italia. Email: sornicol@unina.it

${ }^{1} \mathrm{Si}$ veda il Catalogo delle forme del Corpus OVI dell'italiano antico s. v. conesso; si veda inoltre Tommaseo/Bellini (1929, 2: 517a). Il tipo non è registrato da Battaglia.
} 
Boccaccio, Decameron V, 7 «avvenne che, tornando egli da uccellare e passando lunghesso la camera dove la figliuola gridava, meravigliandosi, subitamente entrò dentro e domandò che questo fosse»

Boccaccio, Esposizioni c. VII (i), par. 98 «E noi, Virgilio e io, in compagnia dell'onde bige, cioè lunghesso l'acque bige, come i compagni vanno l'uno lunghesso l'altro per un cammino» ${ }^{2}$

\section{Sovresso}

Dante, Inferno 23, v. 54 «i piè suoi giunti al letto / del fondo giù, ch'e' furon in sul colle / sovresso noi; ma non lì era sospetto»

Dante, Inferno 34, v. 41 «l'altr'eran due, che s'aggiugnieno a questa / sovresso 'l mezzo di ciascuna spalla, / e sé giugnieno al loco de la cresta»

Dante, Purgatorio 27, v. 23 «E se io / sovresso Gerion ti guidai salvo, / che farò ora presso più a Dio?»

Dante, Purgatorio 31, v. 96 «Tratto m'avea nel fiume infin la gola, / e tirandosi me dietro sen giva / sovresso l'acqua lieve come scola»

Dante, Purgatorio 32, v. 149 «simile mostro visto ancor non fue. / Sicura, quasi rocca in alto monte, / seder sovresso una puttana sciolta / m'apparve con le ciglia intorno pronte»

Dante, Paradiso 19, v. 91 «Quale sovresso il nido si rigira / poi c'ha pasciuti la cicogna i figli» ${ }^{3}$

\section{Sottesso}

Dante, Purgatorio 31, v. 19 «Come balestro frange, quando scocca / da troppa tesa, la sua corda e l'arco / e con men foga l'asta il segno tocca / sì scoppia' io sottesso grave carco»

Boccaccio, Teseida 1.1. (c) «O sorelle Castalie, che nel monte Elicona contente dimorate..., sottesso l'ombra delle fronde amate da Febo»

Livio volg. 5, 26 «Con l'armi e con la virtù è di necessità che... la nazione ribellante, più temerariamente combattente che arditamente, al giogo di sotto, al quale s'è tolta, a ritornare sottesso costrignete» 4

2 Si veda il Catalogo delle forme del Corpus OVI dell'italiano antico, s. v. lunghesso. Il tipo è registrato e descritto da Tommaseo/Bellini (1929, 3: 1304a).

${ }^{3}$ Si veda il Catalogo delle forme del Corpus OVI dell'italiano antico, s. v. sovresso. Si veda inoltre Tommaseo/Bellini (1929, 5: 1051b); Battaglia 19, 647b.

${ }^{4} \mathrm{Si}$ veda il Catalogo delle forme del Corpus OVI dell'italiano antico, s. v. sottesso; Tommaseo/Bellini (1929, 5: 1036c); Battaglia (19: 570a). 
Questi tipi sono stati segnalati da Rohlfs (1949-1954, 2: § 496), che così descrive il processo diacronico alla loro origine: «Esso... dopo alcune preposizioni è divenuto elemento rafforzativo indeclinabile, che ha finito col fondersi con la preposizione». Tuttavia la atrofizzazione del pronome rispetto alla preposizione non si può considerare del tutto completata, dal momento che - come riporta Battaglia (9:292b) - esistono esempi di concordanza del pronome con il sostantivo cui si riferisce:

«Stimarono... acconcio... che, traghettata la Lenza, potesse [la cavalleria] marciare lunghessa sponda del Po» (Siri)

«Otto grosse candele ardevano intorno alla morta, e la cera gocciolava agglomerandosi lunghesse in grosse e bizzarre stalattiti» (Praga)

«Su i pianori dei monti, / lunghesse le vie lapidose» (D’Annunzio)

«Tra quell'ombre che giacciono oziose / sottessa la tranquilla onda lunare, / sognare, o Iole, le passate cose, / i dolci sogni d'un tempo sognare» (Pascoli)

Che il fenomeno sia documentato in scrittori moderni potrebbe essere indizio della conservazione di una più antica condizione strutturale sull'arco di diversi secoli, ma non si può neppure escludere una restaurazione colta di tale condizione, effettuata con l'intento di rendere meno opaca la struttura. Costruzioni come quelle ora riportate, in ogni caso, pongono un interessante problema di descrizione grammaticale: sino a che punto è sostenibile che lunghesso e sottesso, che ancora presentano varianti con flessione governata dai tratti di genere e numero del nome che segue, siano delle vere e proprie preposizioni? Da un punto di vista strutturale, la duplice casistica per cui il dimostrativo è agglutinato con perdita della flessione (tipo lunghesso il fiume), o conserva i suoi tratti morfologici di accordo (tipo lunghesse le vie) può essere rappresentata, rispettivamente con i diagrammi (a) e (b):

(a)

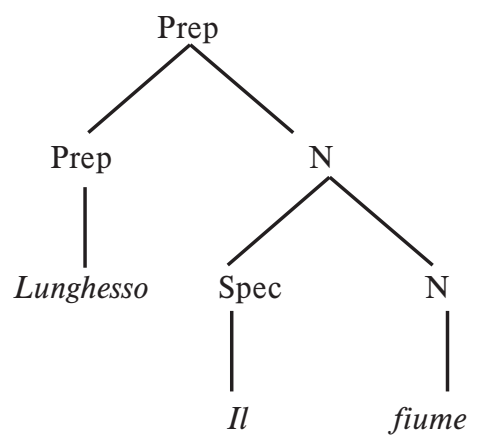


(b)

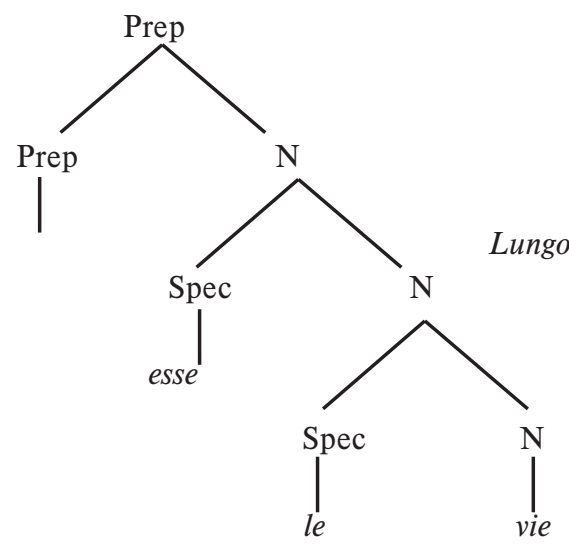

Le due rappresentazioni esplicitano il fatto che, indipendentemente dalle caratteristiche di fonologia frasale (esso appartiene comunque al sintagma intonativo della preposizione), in (a) il dimostrativo esso forma costituente con la preposizione, mentre in (b) forma costituente con il sintagma nominale che segue. Si tratta, come è ovvio, di uno dei casi di possibile asimmetria fra struttura intonazionale e struttura sintattica. Il diagramma (b) mostra che, per costruzioni come lunghesse le vie, non è lecito ricorrere ad un modello di «preposizione flessa», che pure è stato postulato nelle tradizioni grammaticali di varie lingue del mondo. Benché utili per una descrizione configurazionale di massima, le due rappresentazioni tuttavia non esauriscono le molteplici caratteristiche strutturali dei tipi in esame. Come vedremo tra poco, questi ultimi possono avere proprietà semantiche diverse in rapporto ai contesti di occorrenza, e ciò si riflette anche a livello di relazioni sintattiche. Tale situazione mostra che è difficile sistematizzare in una trattazione grammaticale inevitabilmente sincronica la complessità della casistica diacronica.

\section{L'ANALISI SEMANTICA}

Il valore semantico dei tipi preposizionali con ess- in confronto ai rispettivi tipi semplici non si lascia facilmente descrivere. Per quanto riguarda lunghesso, Battaglia (9: 292b) distingue un significato 'lungo, rasente, di fianco, per, attraverso', specialmente in rapporto ad un complemento di moto per luogo reale o figurato, da un significato 'presso, vicino, accanto, a lato', in rapporto ad un complemento di stato in luogo. Il significato 'accanto, a lato' è interpretabile per varie occorrenze della forma nella Commedia, ${ }^{5}$ ma compare anche in scrittori di epoca successiva, ancora nel Novecento, in Pascoli e Pirandello. ${ }^{6}$ Il significato

\footnotetext{
5 Ciò è esplicitato nel Commento di Francesco da Buti a Purg. 2, 10: «Noi, cioè Virgilio et io Dante, eravam lunghesso il mare ancora, cioè allato al mare, che non c'eravamo ancora partiti da esso» (cap. 2, 10-24); a Purg. 19, v. 27: «lunghesso me, cioè allato a me, Dante» (cap. 19, 16-33).

${ }^{6}$ Gli esempi forniti da Battaglia per Pascoli e Pirandello non sembrano sicuramente interpretabili in tal senso.
} 
'a canto, a lato', peraltro, non è esclusivamente in rapporto al tipo con il pronome agglutinato, ma si ritrova già di per sé nella preposizione semplice, che oltre al significato 'accosto, rasente, parallelamente' ha anche quello di 'vicino, accanto, presso': Dante, Vita Nuova cap. 12, par. 1-9) «Avvenne quasi nel mezzo de lo mio dormire che me parve vedere ne la mia camera lungo me sedere uno giovane vestito di bianchissime vestimenta» Battaglia (9: $301 b$, s. v. lungo2). Si tratta di una evoluzione del significato dell'originaria base latina aggettivale LŎNGU- (entrata in funzione nominale in formazioni preposizionali (per longum e per longo)), già attestata in documenti tardo-latini di area italiana. ${ }^{7}$ In ogni caso, rimane il problema di stabilire se lunghesso e lungo siano meri allotropi senza variazione di significato. Le cose stanno diversamente per quanto riguarda la definizione data da Battaglia per il tipo sovresso, 'sopra, proprio sopra', in cui in aggiunta al significato del tipo preposizionale semplice, compare un valore specificativo 'proprio', altrimenti assente Battaglia (19: 647b).

L'esame della tradizione lessicografica più antica non fornisce indizi risolutivi. A proposito di esso in Tommaseo/Bellini (1929, 2: 1370c) si osserva in generale che «si aggiunge talora ad altre voci e preposizioni senza alterar punto il loro significato». Per il tipo conesso si rileva tuttavia che, quando è seguito da pronomi personali, il pronome esso non è un semplice riempitivo, ma figura come «rincalzo». D'altra parte, quando segue un nome, il pronome «è detto per vezzo in vece della semplice preposizione» (Tommaseo/Bellini 1929, 2: 517a). Per lunghesso, il cui significato è reso come 'rasente, accosto', si osserva che «è antica e usata proprietà di linguaggio l'aggiugner [esso] non solamente agli avverbi, ma eziandio a' nomi». ${ }^{8} \mathrm{La}$ «proprietà di linguaggio» è indicata come caratteristica stilistica associabile anche ai tipi sottesso e sovresso. ${ }^{9} \mathrm{Nel}$ complesso, come si vede, la descrizione semantica è tutt'altro che chiara, né sembra più chiaro il sibillino commento stilistico.

\section{CARATTERISTICHE DISTRIBUZIONALI}

Sia l'analisi semantica che quella del processo di grammaticalizzazione richiedono un esame congiunto delle proprietà dei tipi preposizionali italiani qui discussi e di quelle del pronome latino IPSE nei suoi diversi contesti di occorrenza. I tipi italiani mostrano una sequenza rigida Preposizione + ess -+ Pronome obliquo o Preposizione + ess- + Nome, dove il pronome obliquo può essere essere uno della serie personale me, te, se, lui, lei, noi, voi, loro, oppure - limitatamente al tipo conesso - un pronome della serie meco, teco, seco. ${ }^{10}$ In alternativa, il sintagma nominale che segue la struttura Preposizione + ess- può avere come testa un nome proprio o un nome comune.

7 Si veda Arnaldi et alii (1350b). Questo sviluppo di significato si trova anche in numerose forme preposizionali galloromanze antiche e moderne (si veda FEW 5: 407-408).

8 Tommaseo/Bellini (1929, 3: 1304a). Si noti che la caratteristica della «proprietà di linguaggio» era stata chiamata in causa al riguardo già dal Vocabolario degli Accademici della Crusca (quarta impressione), s.v. lunghesso.

9 Per cui si veda in Tommaseo/Bellini (1929, 5: 1036c e 1051b).

10 A proposito della combinazione con meco, teco, seco, in Tommaseo/Bellini (1929, 2: 517a) si parla di un «fiorentinismo», il cui uso eccessivo è da stigmatizzare. 
Quest'ultimo può essere preceduto da un articolo determinato o indeterminato (il che si vede nella maggior parte delle attestazioni) o può essere privo di articolo.

Non tutti i tipi si comportano allo stesso modo per quanto riguarda la frequenza di selezione di un sintagma pronominale o nominale. Con il tipo conesso, la casistica di combinazione con un pronome è infatti più caratteristica, mentre è più rara la combinazione con un nome. ${ }^{11} \mathrm{Si}$ noti, infine, che alcuni tipi preposizionali, come lunghesso e sovresso ammettono costruzione avverbiale: «Tutti i rapporti concorrono ad affermare che Bonaparte è sbarcato non più in Alessandria, ma ad Alessandretta per recarsi di là sull'Eufrate e lunghesso penetrare nel cuore del commercio inglese» (Monti, cit. in Battaglia, 9: 292b). ${ }^{12}$ La struttura in cui il tipo compare in un contesto in cui seguono altri sintagmi preposizionali, riportata da Battaglia (9: 292b), dovrebbe del pari essere considerata avverbiale, con i sintagmi preposizionali successivi analizzabili come apposizioni: Buonarroti il Giovane, «lunghesso verso le case de' Peruzzi» (= 'a lato, verso le case dei Peruzzi').

\section{FUNZIONI DEL DIMOSTRATIVO LATINO IPSE}

La distribuzione ora descritta è in rapporto ad alcune proprietà sintattiche e semantiche del dimostrativo latino IPSE, etimo delle forme italiane ess-. IPSE era usato già in latino classico, ed ancora di più in latino tardo, in un ampio spettro di costruzioni e funzioni pronominali e aggettivali. Quando formava sintagma con un pronome personale o con un nome fungeva da focalizzatore di tale costituente, con valori rappresentabili come 'anche $\mathrm{X}$, proprio $\mathrm{X}, \mathrm{X}$ in persona, $\mathrm{X}$ dal canto suo'. Non è privo di interesse che già in latino post-classico la posizione di IPSE potesse indurre valori semantici diversi. Se IPSE precedeva il costituente pronominale o nominale aveva piuttosto il valore di un attributo di identità (= IDEM): ipse rex 'il re medesimo', se seguiva tale costituente aveva un più spiccato valore di focalizzazione: rex ipse 'il re in persona, proprio il re'.

Un altro valore del dimostrativo latino era quello che possiamo definire «individualizzante», che esprimeva una sorta di iperdeterminazione 'esattamente $\mathrm{X}$ ', talora con una implicazione di esclusione 'e nessun altro'. Il significato di individualizzazione o precisazione di un concetto era specialmente caratteristico di costruzioni preposizionali con valore locativo o temporale: ad ipsum puteum 'proprio vicino al pozzo', in ipsa ciuitate 'proprio nella città', in ipsa hora 'proprio in questo momento, subito'. Costruzioni come queste (specialmente quelle temporali) hanno avuto numerosi continuatori nelle lingue romanze, spesso in espressioni che si sono grammaticalizzate come avverbi. ${ }^{13}$ Un caso particolarmente emblematico è quello dell'avverbio italiano adesso, che deve essere ricondotto ad AD IPSUM (TEMPUS),

11 In Tommaseo/Bellini (1929, 2: 517a) si osserva che quando conesso «non è congiunto a persona» (cioè non si combina con un pronome personale) «è detto per vezzo, invece della semplice preposizione».

12 L'analisi di questo esempio però non sembra incontrovertibile. Non si può escludere infatti che esso sia qui una ripresa anaforica di Eufrate (si veda più avanti, §5).

13 Per una discussione di questo punto, e più in generale delle forme e funzioni di IPSE in latino e della loro sopravvivenza nelle lingue romanze, rinvio a Sornicola (2008), Sornicola (in stampa). 
nonostante le difficoltà fonetiche (la $e$ tonica è aperta e non chiusa, come ci si aspetterebbe). ${ }^{14} \mathrm{Si}$ tratta infatti di un tipo di determinazione di tempo che ha numerosi riscontri in costruzioni avverbiali tra loro affini, presenti in tutta la Romània. È interessante che nel latino di varia epoca la posizione di IPSE nelle costruzioni preposizionali fosse, benché non in maniera esclusiva, soprattutto ante-nominale. Le costruzioni romanze che grammaticalizzano questi tipi, ed in particolare le costruzioni preposizionali di cui ci stiamo qui occupando, testimoniano la prevalenza di tale ordine.

\section{LA GRAMMATICALIZZAZIONE DEI TIPI ITALIANI PREPOSIZIONE + ESSO}

Si pone a questo punto il problema se la trafila di grammaticalizzazione di IPS- sia stata motivata dalle stesse ragioni semantiche in tutti i tipi preposizionali presi in esame. In alcuni contesti i tipi italiani Preposizione + ess- mostrano la conservazione del valore individualizzante 'proprio (in un determinato luogo)'. Come si è detto, tale valore risulta chiaramente esplicitato da Battaglia (19: 647b) per sovresso, definito come 'sopra, proprio sopra'. Non è implausibile pensare che ancora in italiano antico e forse anche in seguito, nella competenza di scrittori e persone di cultura, questo valore di precisazione o iperdeterminazione fosse ancora avvertito. D'altra parte, è possibile che già in Dante, che fa diverse volte ricorso a questi tipi nella Commedia, la scelta fosse, almeno in alcuni casi, indotta da ragioni metriche.

Meno chiare sono le ragioni semantiche che hanno potuto dar vita allo sviluppo del tipo conesso, la cui distribuzione prevalentemente in contesti con pronomi personali potrebbe far ipotizzare una originaria funzione comitativa della costruzione, piuttosto che strumentale. In effetti, si potrebbe qui ravvisare la continuazione e fossilizzazione dei contesti latini in cui IPSE occorreva prima di un pronome personale, come rafforzativo di questo: IPSE EGO 'io stesso, io medesimo', IPSE ILLE 'lui stesso, lui medesimo', e così via. Il principio di fondo all'opera in queste strutture potrebbe dunque essere stata la rideterminazione o ipercaratterizzazione di forme pronominali, un principio attivo in varie lingue del mondo attraverso diversi processi morfologici o sintattici. Bisogna notare peraltro che, specie negli stili non sofisticati del latino, IPSE si può trovare in combinazione con dei pronomi dimostrativi (HIC IPSE, ISTE IPSE, ILLE IPSE). Queste combinazioni hanno dato luogo a tipi romanzi morfologizzati, che hanno mantenuto il valore 'idem' della base IPS(si pensi all'it. stesso, allo sp. ant. eleiso 'medesimo'). Induce a riflessione il fatto che accanto a questi esiti, l'italiano antico abbia anche dei tipi sintagmatici come costoro essi, queste esse (Tommaseo/Bellini 1929, 2: 1370c), a riprova della versatilità e vitalità delle combinazioni di IPS-, testimoniate anche nelle forme dimostrative rafforzate mediante ECCU-

14 Si veda Schuchardt (1891: 240); Cortelazzo/Zolli (1: 20) considerano questa etimologia dubbia. Implausibile l'ipotesi di REW 2558 che pone l'italiano adesso sotto la base DĒ(N)SUS 'dicht'. 
Un ulteriore valore semantico di IPSE, che possiamo definire «possessivo-relazionale», potrebbe trasparire in alcuni contesti in cui compaiono i tipi italiani in esame. Tale significato è antico, e si trova attestato ad esempio in Cicerone, ad Quint. fr. 1, 1,22 «hominis... cum ipsa natura moderati tum... studiis eruditi». Nel Commento alla Commedia, Purgatorio 19, v. 91 («Quale sovresso il nido si rigira / poi c'ha pasciuti la cicogna i figli»), Buti propone una interpretazione che sembra suggerire la permanenza di questa potenzialità della base latina nella competenza linguistica di un uomo del XIV secolo : egli interpreta "Quale sovresso '1 nido, cioè chente e come si gira sopra lo suo nido». È dunque possibile che nelle forme conesso, lunghesso, sovresso, sottesso convergano contesti strutturali e funzioni diverse di IPSE.

Ad ogni modo, quali che siano state le possibili diverse dinamiche semantiche retrostanti ai tipi preposizionali in esame, e quale che sia stata la consapevolezza antica dell'esistenza di determinati valori semantici, che gli scrittori più colti potevano ancora cogliere come continuità con il latino, è indubbio che nell'arco dell'ultimo secolo si sia determinato un processo di opacizzazione totale di queste strutture. Il problema della loro grammaticalizzazione completa come preposizioni in italiano antico lascia perplessi. Non si tratta solo di accertare o meno caratteristiche oggettive e inequivocabili come i tratti di concordanza precedentemente menzionati. L'analisi testuale a più lungo raggio mostra non di rado proprietà della forma esso adiacente alla preposizione che non collimano con l'ipotesi di una sua totale desemantizzazione, condizione necessaria perché si possa ritenere di essere in presenza di forme grammaticalizzate. Così la forma sottesso che occorre nel già citato passo di Livio volg. 5, 256, che qui riporto per comodità: «Con l'armi e con la virtù è di necessità che... la nazione ribellante, più temerariamente combattente che arditamente, al giogo di sotto, al quale s'è tolta, a ritornare sottesso costrignete», rende legittimo ipotizzare che il pronome abbia una funzione anaforica di ripresa del sintagma nominale antecedente giogo, e che pertanto conservi in pieno il suo valore deittico. Tale conservazione conferma che il pronome non è decaduto a mera marca flessiva. Ad una funzione anaforica di ripresa fa pensare anche il già citato contesto di Purgatorio 32, v. 149, che del pari riporto: "simile mostro visto ancor non fue. / Sicura, quasi rocca in alto monte, / seder sovresso una puttana sciolta / m'apparve con le ciglia intorno pronte», dove la forma pronominale sembra coreferente di mostro. Tale uso potrebbe riflettere la continuazione dell'antica funzione dimostrativa di IPSE, ampiamente documentata in italiano antico per le forme libere del pronome / aggettivo esso (si pensi ad esempio a Paradiso 8, 19 «Vid'io in essa luce [= 'in quella luce'] altre lucerne / muoversi in giro»). ${ }^{15}$

C'è poi un altro motivo che a mio avviso complica la tesi della grammaticalizzazione. È emerso in vario modo nella discussione sinora svolta: la possibilità che nella competenza di chi parlava o scriveva in italiano i valori diventati oggi per noi opachi

15 Per questa funzione delle forme libere si veda Tommaseo/Bellini (1929, 2: 1370bss.); Battaglia (5: 427b). Per un esame del valore dimostrativo di IPSE, specialmente in contesti anaforici, rinvio a Sornicola (in stampa). 
fossero invece ancora presenti, sia pure come sfumatura semantica che tuttavia opponeva il tipo preposizionale semplice a quello con esso. Come abbiamo visto, qualche indizio in questa direzione potrebbe essere ravvisato, ed in ogni caso, almeno come principio metodologico, dovremmo lasciare la porta aperta a questa possibilità. La situazione moderna non deve essere proiettata sic et simpliciter su quella antica. A me sembra che il maggior interesse dei tipi conesso, lunghesso, sovresso, sottesso, consista non tanto nel fatto che si possano prestare ad uno studio dei processi di grammaticalizzazione, ma che testimoniano la stretta interpenetrazione e la continuità del volgare rispetto al latino, profondamente riflesse nella sue strutture.

\section{Bibliografia}

ARNALDI, Francesco et alii (2001) Latinitatis italicae medii aevi lexicon: saec. 5. ex. - saec. 11. in. Firenze: Sismel, Edizioni del Galluzzo.

Battaglia, Salvatore (1961-2009) Grande Dizionario della lingua italiana. 21 voll. Torino: Unione Tipografico-Editrice Torinese.

Corpus OVI dell'italiano antico = Corpus testuale del tesoro della lingua italiana delle origini, Istituto Opera del Vocabolario Italiano del CNR, versione online.

Cortelazzo, Manlio/Paolo Zolli (1979-1988) Dizionario etimologico della lingua italiana. 5 voll. Bologna: Zanichelli.

Few $=$ WARTBURG, Walther von (1922-) Französisches Etymologisches Wörterbuch. Bonn: Schröder.

REW $=$ MEYER-LÜBKE, Wilhelm $\left({ }^{3} 1930-1935\right)$ Romanisches Etymologisches Wörterbuch . Heidelberg: Winter.

RoHLFs, Gerhard (1949-1954) Grammatica storica della lingua italiana e dei suoi dialetti. 3 voll. Torino: Einaudi.

SCHUCHARDT, Hugo (1891) «Wortgeschichtliches.» Zeitschrift für romanisches Philologie 15, 237-241.

SoRNICOLA, Rosanna (2008) «Sul problema dei resti di IPSE nella Romània.» In: R. Lazzeroni et alii (a cura di), Diachronica et Synchronica. Studi in onore di Anna Giacalone Ramat. Pisa: Edizioni ETS, 537-562.

Sornicola, Rosanna (in stampa) «Per la storia dei dimostrativi romanzi: i tipi neutri zo, so, ciò e la diacronia dei dimostrativi latini.» Zeitschrift fuer romansiche Philologie.

Tommaseo, Niccolò/Bernardo Bellini (1929) Dizionario della lingua italiana. 6 voll. Torino: Unione Tipografico-Editrice Torinese.

Vocabolario degli Accademici della Crusca. 6 voll. Firenze: appresso Domenico Maria Manni, 1729-1738. 


\section{Riassunto}

\section{TIPI ITALIANI CONESSO, LUNGHESSO, SOVRESSO, SOTTESSO E LA GRAMMATICALIZZAZIONE DI IPSE}

I tipi morfologici caratteristici dell'italiano antico, conesso, lunghesso, sottesso, sovresso, pongono alcuni interessanti problemi di analisi strutturale e di formazione diacronica. Sono possibili due rappresentazioni alternative di struttura in costituenti, una in cui il dimostrativo esso forma sintagma con la preposizione, l'altra in cui forma sintagma con il nome. Le due rappresentazioni potrebbero esplicitare fasi diverse del processo di grammaticalizzazione del dimostrativo nelle costruzioni in esame. Tale processo tuttavia non si lascia facilmente descrivere in maniera unitaria. Decisivo sembra, in ogni caso, l'esame della complessa evoluzione del dimostrativo latino IPSE.

\section{Povzetek}

ITALIJANSKE ZVEZE CONESSO, LUNGHESSO, SOVRESSO, SOTTESSO IN GRAMATIKALIZACIJA ZAIMKA IPSE

Morfološke zveze, značilne za staro italijanščino, conesso, lunghesso, sottesso, sovresso, predstavljajo nekaj zanimivih problemov strukturalne analize in diahronega razvoja. Mogoča sta dva alternativa prikaza strukture po sestavnih elementih; pri prvem kazalni zaimek esso tvori besedno zvezo s predlogom, pri drugem pa s samostalnikom. Ta dva prikaza bi lahko pojasnila različne faze procesa gramatikalizacije kazalnega zaimka v obravnavanih konstrukcijah. Vseeno pa se tega procesa ne da enostavno in enovito opisati. V vsakem primeru se zdi odločilna analiza kompleksnega razvoja latinskega kazalnega zaimka IPSE. 